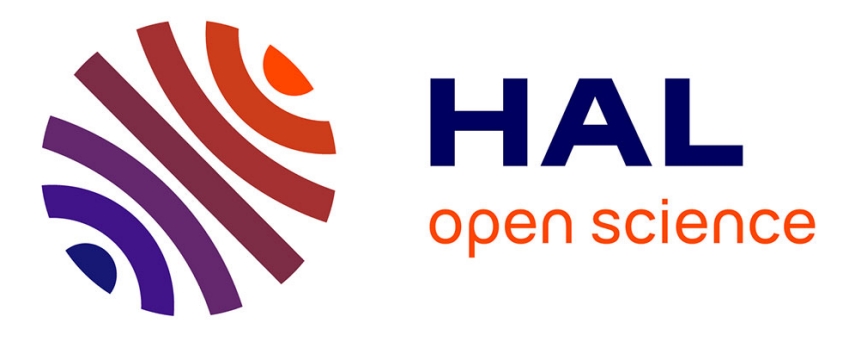

\title{
Robot trajectory generation for three-dimensional flexible load transfer
}

Mohamed Amine Rahmouni, Richard Béarée, Adel Olabi, Mathieu Grossard, Eric Lucet

\section{- To cite this version:}

Mohamed Amine Rahmouni, Richard Béarée, Adel Olabi, Mathieu Grossard, Eric Lucet. Robot trajectory generation for three-dimensional flexible load transfer. IECON 2019, Conference of the IEEE Industrial Electronics Society (IES), Oct 2019, Lisbon, Portugal. cea-03314687

\section{HAL Id: cea-03314687 https://hal-cea.archives-ouvertes.fr/cea-03314687}

Submitted on 5 Aug 2021

HAL is a multi-disciplinary open access archive for the deposit and dissemination of scientific research documents, whether they are published or not. The documents may come from teaching and research institutions in France or abroad, or from public or private research centers.
L'archive ouverte pluridisciplinaire HAL, est destinée au dépôt et à la diffusion de documents scientifiques de niveau recherche, publiés ou non, émanant des établissements d'enseignement et de recherche français ou étrangers, des laboratoires publics ou privés. 


\section{Robot trajectory generation for three-dimensional flexible load transfer}

\author{
Mohamed Amine Rahmouni \\ LISPEN Laboratory \\ Arts et Metiers / CEA tech \\ Lille, France \\ mohamedamine.rahmouni@cea.fr
}

\author{
Richard Bearee \\ LISPEN Laboratory \\ Arts et Metiers \\ Lille, France \\ richard.bearee@ensam.eu
}

\author{
Adel Olabi \\ LISPEN Laboratory \\ Arts et Metiers \\ Lille, France \\ adel.olabi@ensam.eu
}

\author{
Mathieu Grossard \\ Interactive Robotics Laboratory \\ CEA List \\ Gif sur Yvette, France \\ mathieu.grossard@cea.fr
}

\author{
Eric Lucet \\ Interactive Robotics Laboratory \\ CEA List \\ Gif sur Yvette, France \\ eric.lucet@cea.fr
}

\begin{abstract}
This paper addresses the problem of reducing the elastic deformations and the residual vibrations of flexible loads when they are handled by a robot manipulator. During the manipulation of the low-stiffness load, such as bumper or exhaust system in automotive industry, large motion-induced deformations and vibrations may be induced. These deformations will have detrimental effects on the settling time, on the accuracy and on the integrity of the operational process in a constraint environment. The trajectory shaping approaches, i.e smoothing filter or input shaping method are well-known solutions for the suppression of the residual vibration at the end of a rest-to-rest motion. However, using trajectory shaping technique alone may not be sufficient to suppress the static elastic deformations during the transfer phase of the object. Thus, the main contribution of this paper is to propose a two stages feedforward based approach that combines trajectory shaping technique for vibration reduction, with a deformation compensation trajectory. The latter exploits the rotation space of the manipulator to mitigate the flexural motion of the flexible load. The effectiveness of the proposed method is demonstrated by experimental validations on an industrial robot Kuka iiwa.
\end{abstract}

Index Terms-Vibration Suppression, Elastic Deformation, Robot Manipulators, Trajectory Shaping.

\section{INTRODUCTION}

The robotic manipulation is highly concerned with several industrial tasks including assembly operations, loading and unloading parts from an assembly line or a machine and the safe manipulation more specifically in physical HumanRobot Interaction. When handling flexible load at the robot tip, the previous robotics tasks accuracy, sometimes even their feasibility, may be significantly limited by undesired motioninduced vibrations and deformations of the manipulated object.

Many works on flexible objects manipulation have been done in different contexts, such as the clothes manipulation [1]-[3], the ropes manipulation [4] or the transfer of liquid containers with minimal sloshing [5], [6]. The existing methods can be coarsely divided into the state feedback approach and the open-loop approach.
The state feedback approach is generally based on the use of extra force or torque sensors to measure the vibration of the flexible object. In [7], a vibration control scheme is synthesized based on linear feedback of vibration measured by the force sensor. The control is established by adding the vibration control scheme into the controller of the robot wrist joint. In [8], authors propose a velocity field trackingcontrol scheme using proportional integral force feedback to transport and manipulate deformable material with a mobile manipulator. The efficiency of the state feedback approach may be primarily limited by the technical and economic viability of adding extra sensors.

On the open-loop approach side, input shaping technique is a well-established method to suppress motion-induced residual vibrations [9], [10]. A set of finite impulses, called shaper, are convolved with the desired trajectory to suppress or reduce the residual vibration at the end of point-to-point motion. This technique turned to be a robust and effective algorithm in practical applications, where only the natural frequency and the damping ratio of the contributed flexible modes are required [11]. Some of the vibration suppression properties of input shaping technique can be directly found in some trajectory patterns. For instance, in [12], authors propose a finite impulse filter-based trajectory generation taking the vibration reduction into account. Such approach is specifically applied to the jerk-limited trajectory generation in [13], [14]. The jerk time (i.e. the acceleration slope time) can be explicitly linked to the vibration caused by a dominating flexible mode. Hence, the jerk-limited profile can be seen as a special combination of the smoothness property of polynomial profiles and the vibration reduction property of basic input shaping. The main limitation of the open-loop approach is that the vibration can be suppressed at the end of the motion, but the deformation of the flexible object during the motion still exist.

In [15]-[17], the oscillatory behavior of a liquid container during motion is interpreted as a spherical pendulum. Shaping filter are used to suppress the oscillatory behavior of the liquid 
and the trajectory of the robot is adapted by introducing a tilting compensation value to compensate for the deformation during the motion.

Based on the previous combined open-loop approaches, this paper proposes a trajectory generation method for industrial robot manipulator, so that the deformations during threedimensional motion and the residual vibrations of the flexible load are suppressed. The sensitivity analysis of the proposed method regarding the estimation of the dominating modal frequencies is presented. This promising feedforward approach has been experimentally tested on an industrial Kuka LBR iiwa.

\section{LOAD DEFORMATION AND VIBRATION COMPENSATION}

\section{A. Modeling of the load deformations}

In this work, the translational displacement task of a flexible object with a $n$ DOFs serial manipulator $(n \geq 6)$ is investigated. The robot manipulator is kinematically redundant for the previous $3 \mathrm{~d}$ task and among the remaining extra DOFs, the orientation in $3 \mathrm{~d}$ space will be used in the proposed methodology for the elastic deformation compensation. The flexible object to be manipulated is assumed to be submitted to dominating modal deformations. In practice the flexible motion of a nearlinear object, i.e. with a main axis, is dominated by the first bending natural frequency in the two transverse directions. For the sake of simplicity, Fig.1 shows the planar definition of the problem for one bending deformation. One may note that the flexible motion in the other plane can be handled using the same approach. The flexible load is rigidly grasped by the robot gripper at the robot Tool Center Point (TCP). Its position is noted $\mathbf{P}_{t c p}$.

In the following, the elastic deformation of the object is considered. The pure flexural motion of the object tip, noted $w(t)$, is assumed to occur in $Y_{t c p}$ direction only, i.e. no axial deformation is considered. Hence, the coordinates of the object

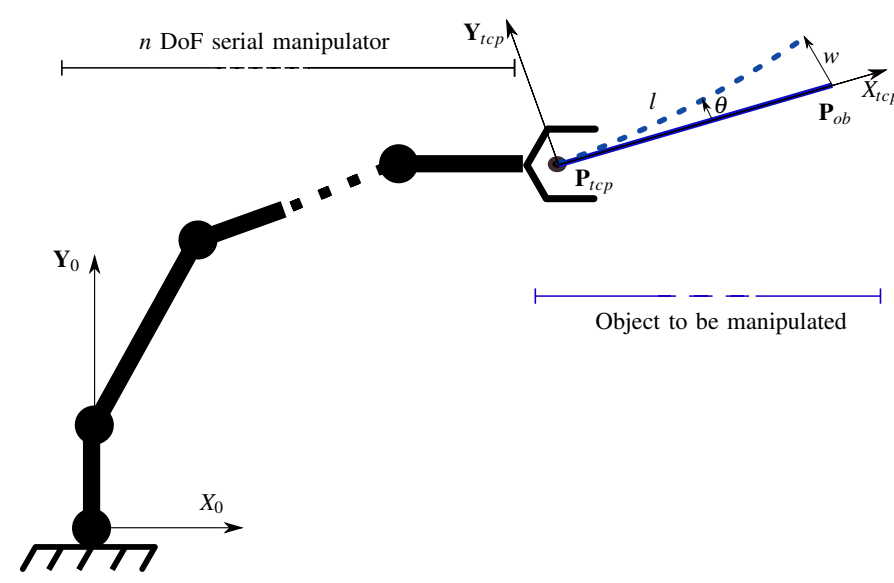

Fig. 1. Parameters definition: robotic manipulation of a cantilevered object with one dominating bending motion. tip, noted $\mathbf{P}_{o b}$, can be expressed in the TCP frame $R_{t c p}$ with the origin point $\mathbf{P}_{t c p}$ as follows

$$
\mathbf{P}_{o b}=\left\{\begin{array}{c}
l \\
w
\end{array}\right\}_{R_{t c p}}
$$

with $l$ and $w$ respectively the length and the flexural deformation of the object. The dynamics of the points $\mathbf{P}_{t c p}$ and $\mathbf{P}_{o b}$ in the fixed absolute frame are submitted to the same rigid motion imposed by the robot TCP trajectory. The evolution of the position $\mathbf{P}_{o b}$ results from the sum of the previous rigid mode with the elastic deformation $w(t)$. Noting $y_{t c p}(t)$ and $y_{o b}(t)$ respectively the position of the points $\mathbf{P}_{t c p}$ and $\mathbf{P}_{o b}$ in $Y_{t c p}$ direction, the deformation $w(t)$ can be written

$$
w(t)=y_{o b}(t)-y_{t c p}(t)
$$

Considering now small variation of the deflection angle, noted $\theta$ in Fig1, the approximated deflection angle of the object can be expressed as

$$
\theta(t)=\frac{w(t)}{l}
$$

The dynamic vibration of the object tip, which occurs in $Y_{t c p}$ direction only, can be modeled by the following general second order equation in continuous Laplace domain

$$
\frac{y_{o b}(s)}{y_{t c p}(s)}=\frac{\omega^{2}}{\omega^{2}+2 \cdot \xi \cdot \omega \cdot s+s^{2}}
$$

with $s$ is the Laplace operator, $\omega$ and $\zeta$ are respectively the natural frequency and the damping ratio of the considered bending mode. Substituting (2) and (3) into (4), the dynamics of the deflection angle $\theta$ can be written

$$
\theta(s)=-\frac{1}{l} \cdot \frac{2 \cdot \xi \cdot \omega \cdot s+s^{2}}{\omega^{2}+2 \cdot \xi \cdot \omega \cdot s+s^{2}} \cdot y_{t c p}(s)
$$

which leads in time domain to the differential equation

$\theta(t)+\frac{2 \cdot \xi}{\omega} \cdot \dot{\theta}(t)+\frac{1}{\omega^{2}} \cdot \ddot{\theta}(t)=-\frac{2 \cdot \xi}{l \cdot \omega} \cdot \dot{y}_{t c p}(t)-\frac{1}{l \cdot \omega^{2}} \cdot \ddot{y}_{t c p}(t)$

Hence, the deflection angle for the considered bending mode will be driven by the acceleration and the velocity of the robot TCP in $\left(Y_{t c p}\right)$ direction. In the same way, the TCP acceleration in $Z_{o b}$ direction may induce vibrations of the first bending mode around $Y_{t c p}$. Assuming that the load vibration can be canceled or reduced, i.e. that the oscillatory behavior given by (6) vanishes and $\ddot{\theta}=\dot{\theta}=0$, the remaining deflection angle would be dominated by the static contribution of (6) given by

$$
\theta(t)=-\frac{2 \cdot \xi}{l \cdot \omega} \cdot \dot{y}_{t c p}(t)-\frac{1}{l \cdot \omega^{2}} \cdot \ddot{y}_{t c p}(t)
$$

Equation (7), which establishes the relation between the static angular deformation during the motion and the instantaneous acceleration and velocity of the robot, is crucial for the proposed methodology. 


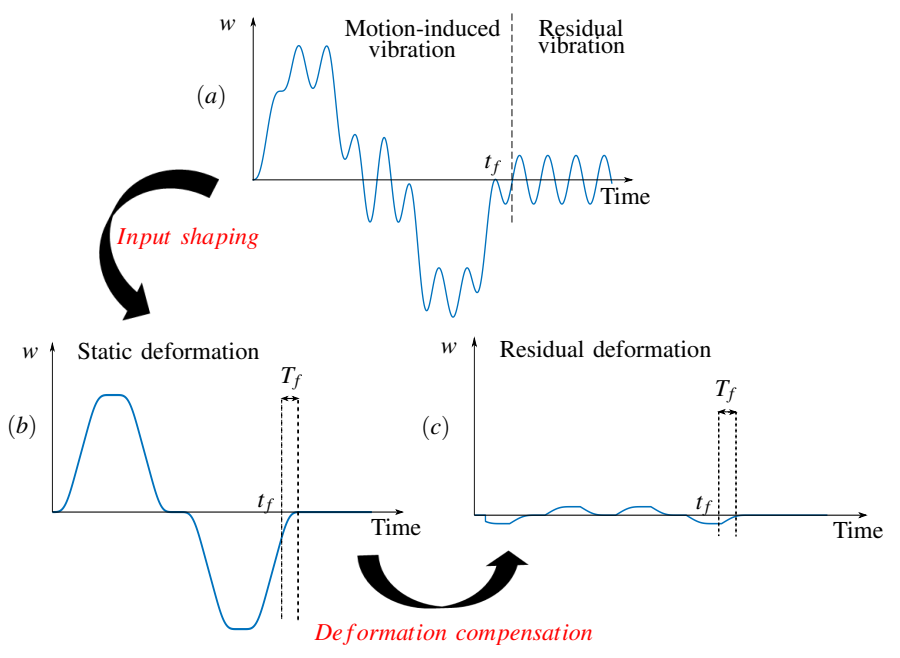

Fig. 2. Deformation $w$ : (a) initial deformation and vibration. (b) deformation with shaping trajectory for vibration compensation. $(c)$ residual deformation with shaping trajectory and deformation compensation.

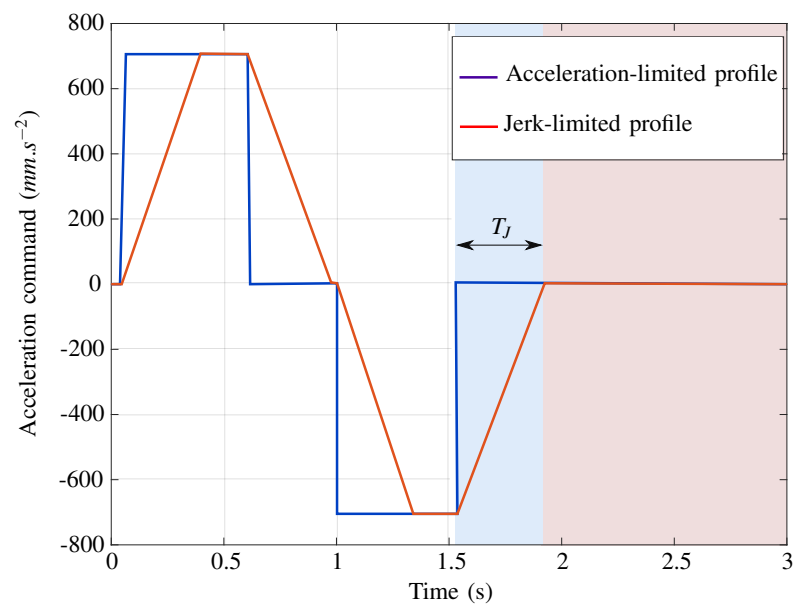

Fig. 3. Example of a jerk-limited profile. The blue zone indicate the time delay due to the shaping filter.

\section{B. Methodology for vibration and deformation compensation}

The proposed methodology, depicted in Figure 2, is a two stages feedforward-based approach. The first stage consists in compensating for the motion-induced vibration via trajectory shaping. The second stage aims at suppressing the remaining static component of the tracking error during the motion, i.e. the deformation of the load tip, using the rotational DOFs of the robot TCP for a proper compensation.

1) First stage - Vibration reduction via trajectory shaping: The dynamic model presented in (6) is a second order system and its excitation force is a combination of the TCP kinematics (acceleration and velocity) along $\left(Y_{o b}\right)$ axis. Trajectory shaping for vibration compensation of such an oscillatory behavior is a well established method. The vibration reduction may be obtained by several types of filters. In this study, the jerk-limited profile, which is available in most robot controllers, is used

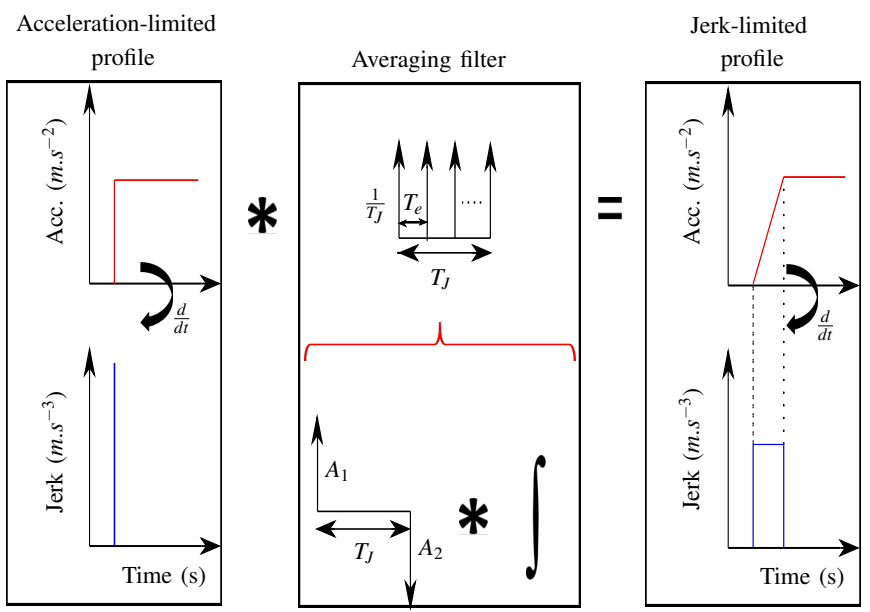

Fig. 4. FIR filtering scheme equivalent to jerk-limited profile. $T_{J}$ is the filter time (equivalent to the jerk time) and $T_{e}$ is the sampling time of the controller.

for the joints trajectory. Fig. 3 describes an acceleration profile for a rest-to-rest motion using Jerk-limited pattern compared to the motion with simple acceleration-limited pattern. The extra motion duration $T_{J}$ compared to the acceleration-limited solution is equal to the jerk time. As depicted in Fig. 4, a Jerklimited trajectory can be easily and efficiently synthesized by applying a moving averaging Finite Impulse Response (FIR) filter, with a filter time equal to $T_{J}$, to an acceleration limited profile. The FIR filter $F_{J L}$ can be described in continuous time domain by the function (8), with $s$ is the Laplace domain operator and $A_{i}$ are the impulse values for the equivalent shaper.

$$
F_{J L}=\frac{1}{s}\left(A_{1}+A_{2} e^{-s T_{J}}\right)
$$

As described in [14], the filter $F_{J L}$ has some common properties with classical shapers. Considering an undamped vibration with a given natural frequency $\omega_{n}$, by setting the filter time $T_{J}$ equals to the natural period time of the vibration, the residual vibration are suppressed. This simple tuning methodology of the FIR filter $F_{J L}$ can be expressed as follows

$$
\begin{aligned}
& T_{J}=k \frac{2 \pi}{\omega_{n}} \\
& A_{1}=-A_{2}=\frac{1}{T_{J}}
\end{aligned}
$$

where $k$ is a positive integer, classically set to one for time saving. A detailed analysis and the adaptation of such a method for the damped vibrations case can be found in [14], [18]. Hence, using the previous feedforward approach, the residual vibrations and most of the vibrations occurring during the motion are suppressed. The next stage consists in suppressing the deformation of the load tip during the motion.

2) Second stage - Residual deformation compensation: Assuming that the load vibration is suppressed by the trajectory shaping method, the residual deflection angle $\theta$ will be given by (7). This remaining motion-induced deformation during the 


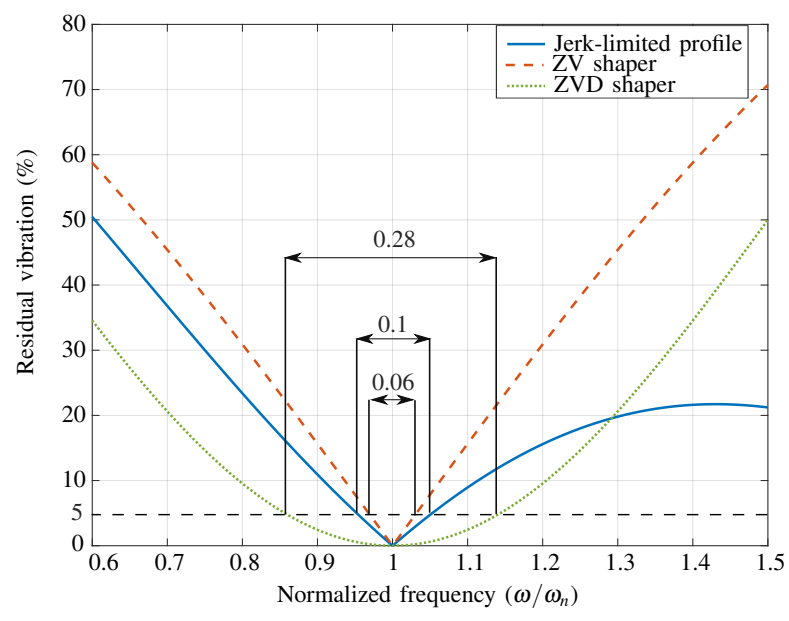

Fig. 5. Sensitivity of the residual vibration suppression action [14].

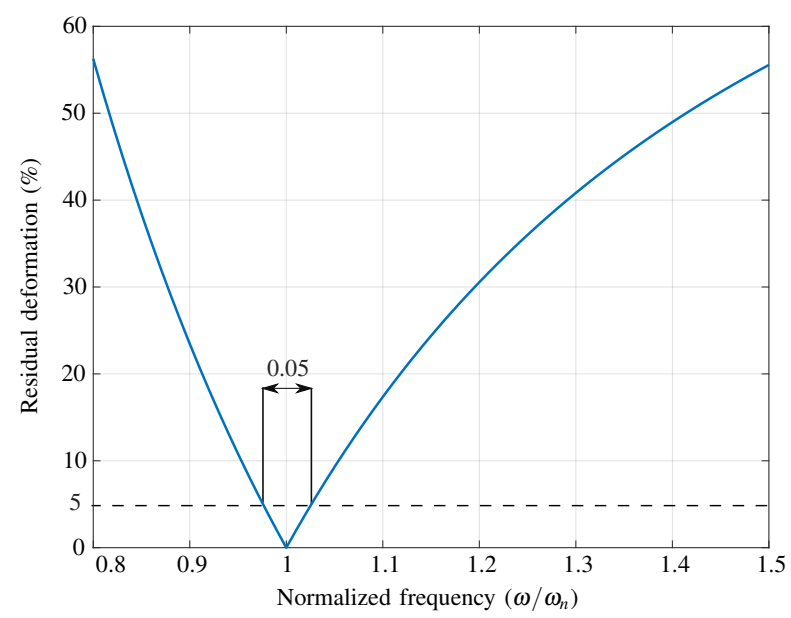

Fig. 6. Sensitivity of the deformation compensation action.

motion can be mitigated by a proper compensation scheme of the deflection angle using the rotational space of the robot TCP, i.e. by adjusting the orientation of the load tip during the motion. This action is added to the design of the feedforward action and can be written

$$
\operatorname{Rot}_{t c p}^{n e w}(\theta(t))=\operatorname{Rot}_{t c p} \cdot \operatorname{Rot}_{c o m p}(-\theta(t))
$$

with $\operatorname{Rot}_{t c p}$ and $\operatorname{Rot}_{\text {comp }}$ are, respectively, the predefined TCP rotation matrix in base frame and the added rotation matrix which counteracts for the estimated deflection angle.

From (7) it is noticed that the angle $\theta$ depends eventually on the TCP acceleration and, to a lesser extent, on the TCP velocity (if the damping is sensitive). Thus, the vibration reduction properties introduced into the TCP trajectory via the shaping method will be extended to the added TCP rotational motion, which will not introduce new vibrations to the load.

\section{Sensitivity analysis}

The proposed feedforward method is inherently sensitive to parameters estimation and variations. Naturally, the main parameter for the vibration and deformation compensation is the value of each modal frequency to be canceled. In input shaping framework, it is customary to define the sensitivity plot, i.e. the percent of residual vibration amplitudes as function of the normalised variation $\left(\omega / \omega_{n}\right)$, with $\omega$ and $\omega_{n}$ respectively the estimated and the real value of the natural frequency. Such a sensitivity plot is depicted in Fig. 5 for the jerk-limited profile, used in this study, and for two wellknown shapers, the Zero-Vibration (ZV) shaper and the ZeroVibration-Derivative (ZVD) shaper (see [10]). Here, 100\% residual vibration corresponds to the vibration induced by an acceleration step command, and robustness or sensitivity is classically defined as the frequency range over which a command induces less than $5 \%$ vibration. These sensitivity curves reveal how much residual vibration will exist when there is an error in the estimation of the system frequency. Hence, the jerk-limited trajectory tune to cancel the residual vibration of one flexible mode will be around $40 \%$ more robust than a $\mathrm{ZV}$ shaper. Moreover, the low-pass filtering property which is introduced by the integrator is effective to reduce significantly the impact of the neglected high frequency modes without any required frequency identification, which is practical for the industrial applications.

The same methodology can be followed to express the sensitivity plot for the deformation compensation. The deformation is affected by the variation of the angle compensation $\theta$ given by (7) and can be expressed as

$$
w_{n}=l \theta=-\frac{2 \xi_{n}}{\omega_{n}} \dot{y}_{t c p}-\frac{1}{\omega_{n}^{2}} \ddot{y}_{t c p}
$$

Where $\omega_{n}$ and $\xi_{n}$ are respectively the frequency and the damping values of the dominant mode in the load vibration. Considering a compensation action with the estimated frequency and damping value $\omega$ and $\xi$, the remaining residual deformation can be expressed as

$$
\frac{\Delta w}{w_{n}}=\frac{w_{n}-w}{w_{n}}=1-\left(\frac{\omega_{n}}{\omega}\right)^{2} \cdot \frac{a \cdot\left(\frac{\omega}{\omega_{n}}\right)+b}{a+b}
$$

Where $a=-2 \cdot \xi \cdot \dot{y}_{o b}, b=-\ddot{y}_{o b} / \omega_{n}$. Assuming now that the damping can be neglected for the dominating deformations, the sensitivity of the deformation compensation is given by

$$
\frac{\Delta w}{w_{n}}=1-\left(\frac{\omega_{n}}{\omega}\right)^{2}
$$

Fig. 6 represents the sensitivity of the deformation compensation according to the normalized frequency. $100 \%$ residual deformation corresponds to the initial deformation without compensation. One can note that the sensitivity to keep the residual deformation below $5 \%$ is almost half the sensitivity of the vibration compensation action. However, in a more practical manner, an error of $10 \%$ for the estimated vibration frequency still allow to reduce the deformation by $80 \%$. 


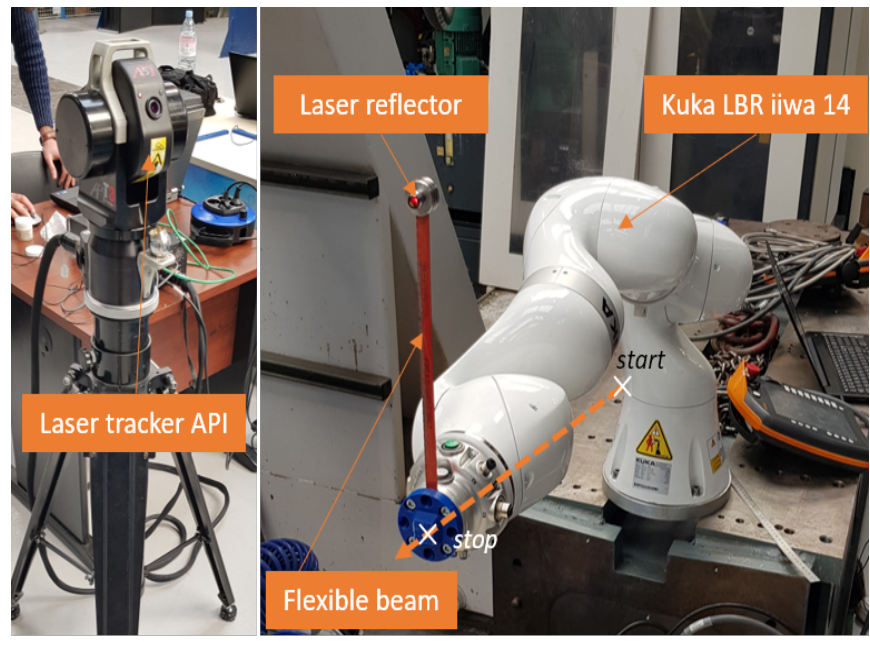

Fig. 7. The experimental setup.

\section{EXPERIMENTAL VALIDATION}

\section{A. Experimental setup}

To validate the proposed methodology and evaluate its effectiveness, an experimental validation has been done with a 7 DOFs robot manipulator kuka iiwa 14, which manipulates a flexible beam (see Fig. 7). A laser tracker from API is used to measure the beam tip displacement along a predefined linear trajectory of the robot TCP. Only the first vibration mode of the flexural motion is considered in the motion direction. The system parameters have been identified as follows: the natural frequency is $\omega=2.2 \mathrm{~Hz}(0.038 \mathrm{rad} / \mathrm{s})$; the beam length is $l=0.25 \mathrm{~m}$; the value of the damping ratio is close to $\xi=0.001$ and therefore the damping can be reasonably neglected for the considered beam.

Four different cases are presented in the following. The first case corresponds to the initial response of the beam with no vibration and deformation compensation. The second experiment consists in adding the deformation compensation action (static compensation) only. In the third case, only the vibration compensation is used and the last case combines the vibration and deformation compensation actions. The different robot TCP trajectories have been generated off-line and then send to the robot joint controller with a sampling time equal to $4 m s$.

\section{B. Discussions}

Fig. 8 shows the evolution of the measured load tip position and Fig. 9 shows the measured position tracking error of the

TABLE I

THE DEFORMATION ERROR COMPARISON IN THE THREE CONSIDERED CASES.

\begin{tabular}{|c|c|c|c|}
\hline Case & $\mathrm{w}_{\max }$ & Vibration (\%) & Deformation (\%) \\
\hline Case 1 - Initial & $12.3 \mathrm{~mm}$ & $100 \%$ (ref.) & $100 \%$ (ref.) \\
\hline Case 3 & $8.8 \mathrm{~mm}$ & $5 \%$ & $71 \%$ \\
\hline Case 4 & $2.3 \mathrm{~mm}$ & $5 \%$ & $18 \%$ \\
\hline
\end{tabular}
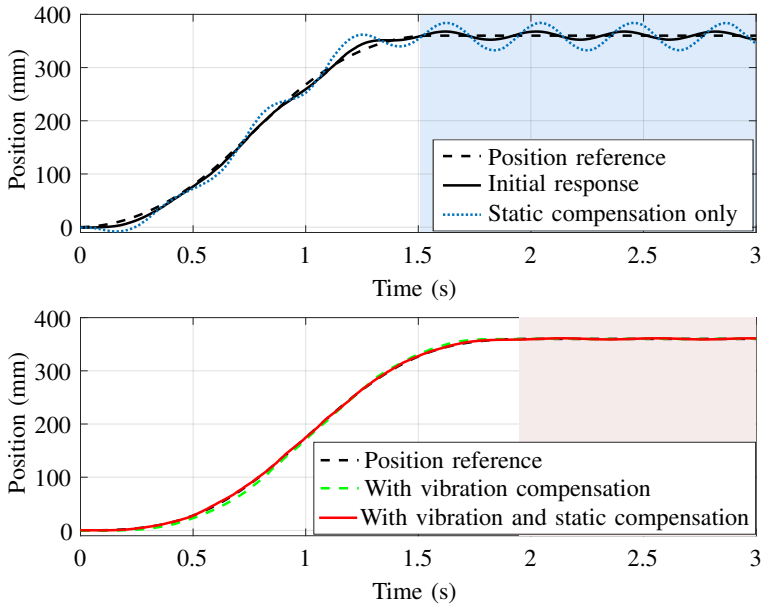

Fig. 8. The load tip position for different cases. The after-motion state of the no-shaped (acceleration-limited) and shaped (jerk-limited) trajectory cases is depicted by the blue and the red zones respectively.

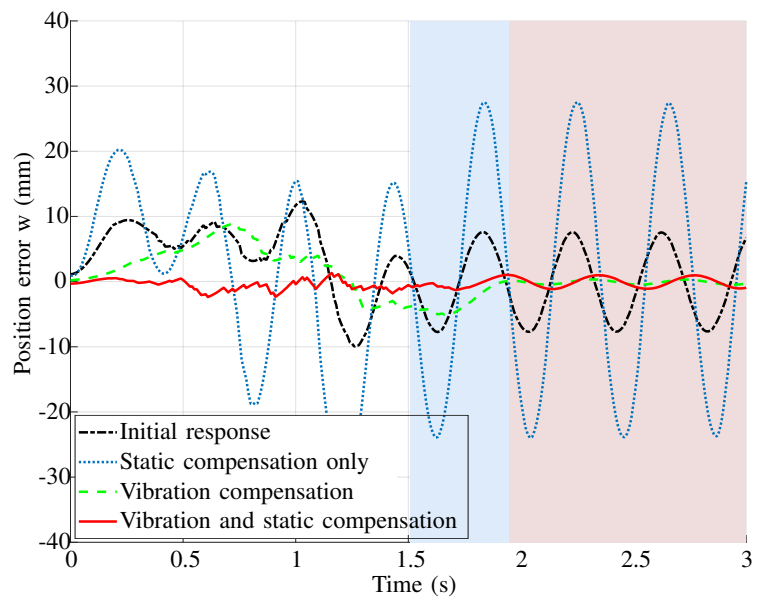

Fig. 9. The load tip tracking error before and after motion. The blue zone indicate the time delay due to the shaping filter for the jerk-limited trajectory.

load tip for the different trajectories. Table I gives the main quantitative results for the different trajectories. For the initial measured response and for the case with the deformation compensation (static compensation) only, the reference trajectory is an acceleration-limited trajectory. For the two other cases, the reference trajectory is the jerk-limited trajectory resulting from the FIR filtering of the acceleration-limited trajectory.

Case 1 - Initial response: The initial response of the load tip is clearly characterized by two different phases. During the motion, the load tip exhibits significant vibrations superimposed to the deformation error pattern. After the motion, the undamped residual vibrations are clearly visible.

Case 2 - Deformation (static) compensation only: In this case, only the angle compensation technique is used. As described in Fig. 9, the vibration amplitude is increased due to the fact that the compensation is done on the unshaped 
acceleration profile. Hence, vibrations induces by the translation motion of the TCP are naturally not suppressed (see (6)), but worse, the added rotation trajectory of the TCP induces new vibrations. For the test trajectory, the contribution of each motion on the vibration level is clearly additive. This case reflects the fact that the proposed deformation compensation cannot be used without a vibration suppression action.

Case 3 - Vibration compensation only: The jerk-limited profile is used in this case to supress the vibrations. Fig. 9 shows that the vibrations are efficiently damped out while the deformation still exists during the motion. The residual vibration amplitude is less than $5 \%$ of the initial one. The remaining small vibration amplitude can be related to different factors such as the estimation bias of the natural frequency and the neglect of the damping effect.

Case 4 - Vibration and deformation compensation: This case shows that the proposed two-stages feedforward method is efficient to significantly reduce the vibrations and the deformation. The residual tracking error of the load tip is less than $18 \%$ and the residual vibration value is equivalent to the previous case.

\section{CONCLUSION}

In this study, we proposed a feedforward strategy to compensate for the deformation and vibrations during the translational motion of a flexible load with a robot manipulator. The method is based on the combination of a trajectory shaping stage for the vibration suppression, with a trajectory compensation scheme for the elastic deformation occurring during the motion. The latter action makes use of the rotation space of the robot manipulator, which is not used for the task. The sensitivity of the two actions with regard to the estimated vibration frequencies has been derived. The experimental tests, which has been conduct using a Kuka iiwa robot and a pure flexible beam, demonstrated the effectiveness of the proposed approach. Future works will focus on the generalization of the methodology for translational and rotational motion of the flexible load.

\section{ACKNOWLEDGMENT}

The author would like to thank the "European Union" and the french region "Hauts de France" for the financial support.

\section{REFERENCES}

[1] Z. Pan and D. Manocha, "Motion planning for fluid manipulation using simplied dynamics," in IEEE/RSJ International Conference on Intelligent Robots and Systems. IEEE, 2016.

[2] Y. Li, D. Xu, Y. Yue, Y. Wang, S.-F. Chang, E. Grinspun, and P. K. Allen, "Regrasping and unfolding of garments using predictive thin shell modeling," in Robotics and Automation (ICRA), 2015 IEEE International Conference on. IEEE, 2015, pp. 1382-1388.

[3] Y. Li, Y. Wang, Y. Yue, D. Xu, M. Case, S. F. Chang, E. Grinspun, and P. K. Allen, "Model-driven feedforward prediction for manipulation of deformable objects," IEEE Transactions on Automation Science and Engineering, 2018, vol. PP, no. 99, pp. 1-18.

[4] S. H. Huang, J. Pan, G. Mulcaire, and P. Abbeel, "Leveraging appearance priors in non-rigid registration, with application to manipulation of deformable objects," in Intelligent Robots and Systems (IROS), 2015 IEEE/RSJ International Conference on. IEEE, 2015, pp. 878-885.
[5] W. Aribowo, T. Yamashita, K. Terashima, H. Kitagawa, "Input shaping control to suppress sloshing on liquid container transfer using multi-joint robot arm," in: Intelligent Robots and Systems (IROS), 2010 IEEE/RSJ International Conference on, 2010, pp. 3489-3494.

[6] W. Aribowo, T. Yamashita, K. Terashima, "Integrated trajectory planning and sloshing suppression for three-dimensional motion of liquid container transfer robot arm," Journal of Robotics, 2015.

[7] Z. Jiang and M. Kohno, "Robotic manipulation of exible objects based on vibration control using force sensors," Systems, Man and Cybernetics, 2002 IEEE International Conference on, vol. 4, 2002.

[8] Tanner H. "Mobile manipulation of flexible objects under deformation constraints," IEEE Transactions on Robotics, 2006,pp. 179-184.

[9] Singer, N., and Seering, W.. "Preshaping Command Inputs to Reduce System Vibration," Journal of Dynamic, 1990.

[10] W. Singhose, "Command shaping for flexible systems: A review of the first 50 years," International Journal of Precision Engineering and Manufacturing, 10(4), pp. 153-168.

[11] W. Singhose, and L. Pao, "A comparison of input shaping and timeoptimal flexible-body control," Control Engineering Practice, 2012, 5(4), pp. $459-467$.

[12] L. Biagiotti, and C. Melchiorri, "FIR filters for online trajectory planning with time- and frequency-domain specifications," Control Engineering Practice, 2012, 20(12), pp. 1385-1399.

[13] R. Bearee, A. Olabi, "Dissociated jerk-limited trajectory applied to timevarying vibration reduction". Robotics and Computer-Integrated Manufacturing, 2013. 29 pp. 444-453.

[14] R. Bearee, "New Damped-Jerk trajectory for vibration reduction,"Control Eng. Pract., 2014, vol. 28, pp. 112-120.

[15] L. Moriello, L. Biagiotti, C. Melchiorri, and A. Paoli, "Manipulating liquids with robots: A sloshing-free solution," Control Engineering Practice, 2018, vol. 78, pp. 129-141.

[16] S. J. Chen, B. Hein, H. Worn, "Using acceleration compensation to reduce liquid surface oscillation during a high speed transfer", IEEE International Conference on Robotics and Automation, 2007, pp.29512956.

[17] J. T. Feddema, C. R. Dohrmann, G. G. Parker, R. D. Robinett, V. J. Romero, D. J. Schmitt, "Control for slosh-free motion of an open container," IEEE Control Systems 17 (1), (1997), pp. 29-36.

[18] L. Biagiotti, C. Melchiorri and L. Moriello, "Optimal Trajectories for Vibration Reduction Based on Exponential Filters," IEEE Trans. on Control Syst. Tech., 24 (2), pp. 609-622, 2016. 